\title{
Uso de infiltrante resinoso (ICON) para tratamento de lesões de mancha branca
}

\author{
Use of resinous infiltrant (ICON) for the treatment of white stain injuries
}

\author{
Danieli Dias" $"$
}

UNILESTE, Ipatinga, Minas Gerais, Brasil. *Autor para correspondência. E-mail: danielidias1515@gmail.com

\begin{abstract}
Resumo. Introdução: O Infiltrante Resinoso (ICON) preenche as microporosidades do corpo da lesão de mancha branca, pela infiltração de uma resina de baixa viscosidade fotopolimerizável, afim de camuflar as manchas brancas, melhorar a estética e impedir a progressão da cárie dental. Revisão: Alterações morfológicas de mancha branca, tanto de origem préeruptiva (fluorose, amelogênese imperfeita), quanto, pós-eruptiva (lesão de cárie), causa dispersão dos raios de luz incidentes, devido à diferença do índice de refração da hidroxiapatita e do ar, que preenche os poros desmineralizados e resulta nesse efeito óptico inestético. No entanto, o infiltrante resinoso ICON, que através de forças capilares é transportado nas porosidades do esmalte, onde preenche os espaços vazios, e após sua polimerização, bloqueia as vias de difusão para ácidos cariogênicos e minerais dissolvidos, o que proporciona paralisação e alteração da dispersão da luz pelo índice de refração (IR) da resina, assemelhando-se ao esmalte saudável existente, ou seja, camuflando a mancha branca. Discussão: A função estética, mimetização da lesão de mancha branca, susceptibilidade de pigmentação e funcional microdureza do esmalte, estão diretamente relacionadas à profundidade e extensão das lesões, tendo em vista que, sua rugosidade é aceitável. Considerações finais: O infiltrante resinoso (ICON), camufla as lesões de mancha branca superficiais, aumenta a microdureza do esmalte e paralisa a atividade cariosa.
\end{abstract}

Palavras-chaves: esmalte dentário, infiltrante resinoso, mancha branca.

\begin{abstract}
Introduction: The Resin Infiltrant (ICON) fills the microporosity of the white spot lesion body, by infiltrating a light-curing low viscosity resin, in order to camouflage white spots, improve aesthetics and prevent the progression of dental caries. Review: Morphological alterations of white spot, both pre-eruptive (fluorosis, amelogenesis imperfecta) and post-eruptive (caries lesion), cause dispersion of incident light rays, due to the difference in the refractive index of hydroxyapatite and of air, which fills the demineralized pores and results in this unsightly optical effect. However, the resinous infiltrant ICON, which through capillary forces is transported in the porosities of the enamel, where it fills the empty spaces, and after its polymerization, blocks the diffusion pathways for cariogenic acids and dissolved minerals, which provides paralysis and alteration of the light scattering by the refractive index (RI) of the resin, resembling the existing healthy enamel, that is, camouflaging the white spot. Discussion: The esthetic function, mimicry of the white spot lesion, pigmentation susceptibility and functional microhardness of the enamel are directly related to the depth and extension of the lesions, considering that its roughness is acceptable. Final considerations: The resinous infiltrate (ICON) camouflages superficial white spot lesions, increases enamel microhardness and paralyzes carious activity.
\end{abstract}

Keywords: dental enamel, resinous infiltrate, white spot.

\section{Introdução}

O esmalte dentário é formado por ameloblastos, células muito sensíveis a alterações sistêmicas e originadas na lâmina dentária do epitélio oral. Ademais, o esmalte é considerado o tecido mais duro do corpo humano, sendo constituído de $97 \%$ de componentes inorgânicos, 3\% de matéria orgânica, $2 \%$ de proteínas não colágenas e 1\% de água (Katchburian \& Arana, 1996)

Além disso, as anormalidades do esmalte em sua formação serão visíveis frequentemente na superfície dos dentes, pois não ocorre remodelação deste tecido após o início da sua composição (Neville \& Damm, 2009).

As lesões de mancha branca ocorrem devido à alteração pré-eruptiva, por um defeito de desenvolvimento do esmalte, quando o fator causal atuar durante a formação do mesmo ou pós-eruptivas, quando provocadas por um agente externo causando uma desmineralização sob a forma de uma lesão não-cavitada (Torres \& Borges, 2015; Borges et al., 2017; Mazur et al., 2018 apud Roselli, 2019). 
Outrossim, as lesões de mancha branca são assim chamadas devido a sua aparência branca e opaca e podem ser observadas clinicamente quando atingem uma profundidade de lesão acima de 300/ 500 micrometros a partir da superfície do esmalte (Min et al., 2015).

Nesse sentido, a mancha branca, ocorre devido aos diferentes índices de refração da hidroxiapatita (1,62$1,65)$ e do ar $(1,0)$ que preenche os poros que foram desmineralizados. No entanto, este efeito óptico acarreta o comprometimento estético dos dentes anteriores (Oguro et al., 2016).

Sendo a mancha branca manifestação clínica de diversas patologias é imprescindível um diagnóstico individualizado para escolha do tratamento. No contexto de odontologia minimamente invasiva, que visa à preservação da estrutura dental sadia e prevenção, surge no mercado os infiltrantes resinosos.

O mecanismo dos infiltrantes resinosos envolve forças capilares para transportá-los nas porosidades do esmalte e, após sua polimerização, bloquear as vias de difusão para ácidos cariogênicos e minerais dissolvidos (Paris et al., 2007). Esta técnica objetiva mascarar lesões de esmalte causadas por várias etiologias sem sacrificar o tecido saudável, permitindo melhorar esteticamente a aparência dos dentes anteriores (Mazur et al., 2018).

\section{Revisão}

O esmalte dentário é formado pelos ameloblastos originados da lâmina dentária do epitélio oral, que expressam um importante conjunto de genes que codificam a produção de proteínas essenciais para a formação do tecido dentário (Moffatt et al., 2008).

A amelogênese, processo de formação do esmalte, é dividida em cinco fases: morfogenética, diferenciação, secretora, maturação, protetora, respectivamente. Na fase morfogenética ocorre multiplicação das células do epitélio interno e iniciação da fase de campânula. Já na fase de diferenciação ocorre indução da diferenciação das células da papila dentário, pelos pré-ameloblastos e alongamento das células do epitélio interno do esmalte (Bath-Balogh \& Fehrenbach, 2012).

Em seguida, na fase secretora ocorre secreção da matriz orgânica sobre a dentina do manto. Na fase de maturação apresenta formação dos prismas do esmalte e deposição de proteínas como as amelogeninas, tuftelinas, ameloblastinas e metaloproteínas. E por último na fase protetora, os ameloblastos perdem sua polaridade e formam o epitélio reduzido que recobre o esmalte maduro até a erupção do dente, quando é rompido (Mangueira et al., 2011).

Nesse sentido, Gerlanch et al. (2000) relataram que alterações nutricionais, distúrbios de perfusão vascular, bem como distúrbios no metabolismo de cálcio levam a alterações do esmalte. Visto que, o esmalte dental é formado por células epiteliais com grande síntese proteica e extremamente sensíveis a alterações sistêmicas.

O dente quando irrompe na cavidade bucal, o esmalte está totalmente mineralizado. Sua constituição é de 96\% de material mineral e 4\% de material orgânico e água (Sharawy \& Yaeger, 1989). Em decorrência da sua composição é considerado o tecido mais mineralizado e duro do corpo humano, tendo como protagonista da sua composição inorgânica a apatita.

Além disso, devido a organização dos cristais de hidroxiapatita, variante da apatita, e o índice de refração da luz o esmalte dentário apresenta-se translúcido. Desse modo, quando há uma mudança na estrutura mineral do tecido de origem pré-eruptiva (fluorose), ou pós-eruptiva (lesão de cárie), ocorre uma dispersão dos raios de luz incidentes, devido à diferença do índice de refração, acarretando o comprometimento estético do dente.

No entanto, muitas patologias culminam em alterações estéticas do esmalte, devido à aparição de mancha branca na superfície dental, dentre elas destacam-se: fluorose dentária, cárie e amelogênese imperfeita.

\section{Fluorose}

Fluorose dentária, ocorre pela deficiência na mineralização do esmalte devida à ingestão diária de fluoretos durante o desenvolvimento dental (Aoba \& Fejerskov, 2002). No processo de formação da fluorose ocorre depósito de flúor na estrutura dentária, levando a alterações nos ameloblastos, modificações na homeostase do cálcio e na formação dos cristais de apatita (Fejerskov, Thylstrup \& Larsen, 1977). Em detrimento disso, ocorre deficiência na mineralização do esmalte, o que o torna mais poroso e comprometido esteticamente.

Contudo, é impossível prever o aparecimento e/ou severidade da fluorose observando apenas a quantidade de flúor ingerida durante a formação do esmalte, pois, apenas o flúor absorvido tem potencial de causar fluorose. Nessa perspectiva, pode-se afirmar que a severidade e a distribuição da fluorose dependem da concentração e duração da exposição do flúor, do estágio de atividade do ameloblastos e da suscetibilidade 
individual, atingindo de maneira mais severa os dentes permanentes do que os decíduos, quando ambos estão expostos à mesma concentração de flúor (Campos, 1998).

Por conseguinte, é descrito que além da dosagem outros fatores interferem na severidade da doença: taxa de crescimento esquelético, baixo peso corporal e períodos de remodelamento ósseo constituem-se fases de maior absorção do flúor, estado nutricional e da homeostase do cálcio também são fatores relevantes (Denbesten, 1999).

\section{Cárie dental associada à aparelhos ortodônticos fixos}

Cárie dentária é uma doença multifatorial, infecciosa, transmissível e dieta dependente, que produz uma desmineralização das estruturas dentárias (Fitzgerald \& Keyes, 1960; Keyes, 1960 apud Lima, 2007). Nesse sentido Guedes Pinto (2016) diz que alguns fatores podem influenciar no desenvolvimento da cárie dental, dentre eles destacam-se: fatores primários, hospedeiros, dieta, dente, saliva, flora e os fatores secundários comportamentais e socioeconômicos.

No entanto, temos a placa bacteriana como fator etiológico da doença cárie, no qual se manifesta inicialmente na superfície dental como uma lesão de mancha branca, resultado da liberação de ácidos provenientes da metabolização de açucares, que causam a dissolução do esmalte. No mais, Marsh (1995) definiu biofilme (placa bacteriana) como uma cultura mista, correspondendo a uma comunidade microbiana tridimensional formada numa interface que se pode tornar espacialmente heterogênea, devido aos gradientes físico-químico que se desenvolvem no seu interior.

Os aparelhos ortodônticos fixos, devido ao elevado número de acessórios colocados na superfície dentária, aumentam o número das áreas de retenção de biofilme, pois dificultam a higienização, que por sua vez, auxilia na maturação do biofilme e a desmineralização do esmalte dentário (Newman, 2007). Desse modo, a maturação do biofilme contribui para a proliferação de bactérias na cavidade bucal, uma vez que, eleva a quantidade de hidratos de carbono, possibilitando colonização de lactobacilos e streptococus mutans, principais bactérias causadoras da cárie dental.

A lesão cariosa ocorre pelo desequilíbrio do fenômeno des-re, que, por sua vez, é desencadeado por ácidos provenientes das bactérias do biofilme no processo de fermentação dos carboidratos. Tendo por consequência a queda do $\mathrm{PH}$ bucal e perda de íons de cálcio $(\mathrm{Ca})$ e fosfato para o meio bucal.

Diante desta desmineralização frequente, a saliva por meio da capacidade tampão, não consegue neutralizar a cavidade oral, ou seja, reparar a perda de minerais, que resulta a lesão de mancha branca.

Øgaard (2008), diz que o desenvolvimento da Lesão de Mancha Branca (LMB), um processo muito rápido, quando o paciente não faz uso de fluoretos, tornar-se visível em torno dos suportes ortodônticos (braquetes) em um mês, período equivalente de uma consulta e outra.

Ademais, a chamada lesão branca do esmalte é a primeira manifestação clínica da cárie dentária e resulta da desmineralização subsuperficial da área afetada. Esta tonalidade da lesão resulta da perda de mineral, que altera o índice de refração relativo ao esmalte normal (Melo, Teixeira, \& Domingues, 2006).

Até o momento vários estudos foram realizados com intuito de evidenciar a prevalência de lesão de mancha branca em pacientes que apresentam aparelho ortodôntico fixo, as fases, os dentes mais acometidos e a idade mais susceptível, vejamos:

Bishara e Ostby (2008): "Pacientes ortodônticos apresentam significativamente mais LMB quando comparados a pacientes não ortodônticos, e essa alta incidência foi relatada em diversos estudos".

Akin et al. (2013): "Após o tratamento ortodôntico fixo, 65\% dos pacientes apresentavam LMB, e desse total de pacientes $35 \%$ apresentavam LMB leve, $25 \%$ LMB grave e $5 \%$ apresentavam cavitação".

Benson et al. (2005) e Willmot (2008) "Geralmente a LMB ocorre na face livre dos dentes, fato incomum em pacientes sem aparelho".

Dias (2015): "Pré-adolescentes apresentam maior risco de desenvolver lesão de mancha branca (LMB) nos dentes ântero-superiores”.

Chapman et al. (2010): "A gravidade das lesões de mancha branca (LMB) é maior em pacientes que começaram tratamento na pré-adolescência em relação aqueles que iniciaram no final da adolescência”.

Diante do exposto, nota-se que pacientes ortodônticos, principalmente adolescentes, apresentam alto índice de cárie ativa, que se manifestam inicialmente através da mancha branca em volta dos bráquetes ortodônticos, na face livre (vestibular) dos dentes, área estética.

\section{Amelogênese imperfeita}

Amelogênese imperfeita trata-se de um distúrbio exclusivamente ectodérmico, pois os componentes mesodérmicos do dente, que constituem a dentina e a polpa, encontram-se normais. Sendo assim, esta 
patogenia não está vinculada com quaisquer defeitos generalizados, pois é constituída por um conjunto defeitos hereditários do esmalte (Bevilacqua, Sacramento, \& Felício, 2010).

Desse modo, a amelogênese imperfeita manifestam-se de três tipos distintos: hipoplásico, à deficiência de matriz; hipomineralizado, déficit na calcificação; e hipomaturado, defeito na maturação dos cristais de esmalte.

Nesse sentido, a amelogênese é classificada de acordo com as características clínicas: Na hipoplásica, não existe a matriz de esmalte adequada formada, o esmalte pode ter pouca espessura e/ou fossas e caneletas. Já na hipocalcificada, a matriz tem espessura normal, sua calcificação é deficiente e clinicamente exibe um esmalte não resistente, opaco, branco amarelado e sofre alterações em sua estrutura (Ainamo \& Cutress, 1982; Ruschel et al., 2001 apud Passos et al., 2007). No caso do hipomaturado, a matriz do esmalte é depositada adequadamente e começa a mineralizar, mas, há um defeito na maturação da estrutura de cristais do esmalte. Os dentes afetados apresentam coloração opaca-amarelada, esmalte amolecido e quebradiço, mas não exibe alterações na forma (Neville \& Damm, 2009)

Esse distúrbio ectodérmico não possibilita um tratamento preventivo, por apresentarem origem genética, nesse caso, os tratamentos iniciam-se depois da patogenia instalada.

Visto que procedimentos mais invasivos, nem sempre são a melhor escolha, surge a odontologia minimamente invasiva, no qual há máxima preservação da estrutura dental, abordagem de promoção de saúde e quebra de paradigmas quanto a filosofia do modelo mecanicista tradicional (Fejerskov \& Kidd, 2011; Degrange \& Roulet, 1997; Ericson, 2004; Ericson et al., 2003 apud Tumenas et al., 2014).

Tomando por base os princípios de preservação do tecido dentário, em 1975 surge um dos primeiros relatos da infiltração de materiais em lesões de mancha branca. Davila et al. (1975), realizaram infiltração de um adesivo comercial em lesões naturais e artificiais em dentes humanos com ou sem condicionamento ácido fosfórico $50 \%$. Concluíram que o condicionamento é necessário, uma vez que remove a camada mais externa do esmalte, e afirmam que a técnica é efetiva na paralisação das lesões de mancha branca. Nomearam este procedimento como "plastificação".

Todavia, no final dos anos 2000, através de estudos realizados na Charité University Hospital de Berlim e na Kiel University, o infiltrante resinoso (ICON), constituído por três seringas: Icon-Etch ${ }^{\circledR}$ (ácido clorídrico), Icon-Dry ${ }^{\circledR}$ (etanol) e Icon-Infiltrant ${ }^{\circledR}$ (resina infiltrante), passou ser comercializado (Roselli, 2019).

No entanto, o mecanismo dos infiltrantes resinosos envolve forças capilares para transportá-los nas porosidades do esmalte e, após sua polimerização, bloquear as vias de difusão para ácidos cariogênicos e minerais dissolvidos (Paris et al., 2007). O método de infiltração de resina é baseado na difusão em profundidade, na capacidade do infiltrante resinoso preencher os espaços vazios induzido pela desmineralização e na modificação do aspecto do dente lesado, que ocorre devido a alterações na dispersão da luz pelo índice de refração (IR) da resina, que camufla a lesão de mancha branca, deixando-a e semelhante ao esmalte saudável existente (Tirlet, Chabouis, \& Attal, 2013; Denis et al., 2013; Mazur et al., 2018; Attal et al., 2014 apud Roselli, 2019).

Entretanto, o objetivo do infiltrante resinoso (ICON) é paralisar lesões incipientes, em faces proximais e livres tampando os poros da lesão e interferindo na ação dos ácidos (Paris et al., 2007). Assim, a infiltração desse material cria uma barreira de difusão dentro da lesão de mancha branca impedindo sua progressão e fortalecendo a estrutura de esmalte afetado (Correia, 2012). Nesse sentido, Ulrich et al. (2015) diz que o infiltrante resinoso vai encapsular os prismas de esmalte fortalecendo as estruturas do esmalte e evitando a cavitação.

No entanto, as áreas opacas afetadas, quando tratadas pela resina de baixa viscosidade, tendem a assumir um aspecto semelhante aquele do esmalte normal e saudável circundante (Thylstrup \& Fejerskov, 1978) retomando o aspecto estético (Meyer-Luckel \& Paris, 2009).

Sendo assim, está técnica de infiltração com resina fluída pode ser uma opção válida para camuflar lesões de esmalte causadas por várias etiologias sem sacrificar o tecido dura saudável, permitindo melhorar esteticamente a aparência dos dentes anteriores (Mazur et al., 2018).

O efeito de mascaramento da cor, é um fenômeno óptico, que ocorre devido ao índice de refração do infiltrante, 1,52, ser próximo do índice do esmalte saudável, 1,62, quando comparado com o índice de refração da água e ar (Paris \& Meyer-Lueckel, 2009; Torres et al., 2011 apud Russi, 2019).

Quando comparada com outros produtos como, compostos fluoretados, o infiltrante resinoso (ICON) é vantajoso devido à sua capacidade de penetração, que estabiliza mecanicamente a estrutura do esmalte poroso e por impedir o fornecimento de nutrientes para as bactérias cariogênicas (Rahiotis et al., 2015). Diferentemente dos compostos fluoretados, que criam uma barreira apenas superficial no esmalte, os 
infiltrantes resinosos criam uma barreira tanto na superfície quanto no interior da lesão e isso gera o reforço da estrutura desmineralizada com uma matriz resinosa polimerizada (Torres et al., 2012).

Ademais, segundo Takashino et al. (2016) a infiltração com Infiltrante Resinoso (ICON) demonstrou uma proteção quase perfeita da desmineralização do esmalte contra o ataque ácido e a redução percentual foi calculada em $95,4 \%$.

Contudo, o Infiltrante Resinoso (ICON) preenche as microporosidades do corpo da lesão pela infiltração de uma resina de baixa viscosidade fotopolimerizável, otimizada para proporcionar uma rápida penetração nas lacunas do esmalte dental afetado (Tong et al., 1993). A fim de, mascarar as manchas brancas, ou seja, melhorar a estética, impedir a progressão da cárie e fortalecer a estrutura de esmalte afetado.

\section{Discussão}

O Infiltrante resinoso (ICON), possui como princípio a odontologia minimamente invasiva, sendo, crescente sua utilização nos consultórios para tratamento de lesões de mancha branca. No entanto, há muitas controvérsias sobre o produto.

Tomando por base os objetivos do Infiltrante Resinoso (ICON), surgem algumas especulações quanto a microdureza do esmalte (fortalecimento da estrutura do esmalte afetado) e a textura da superfície e aparência final (estética) após o uso do produto. Vejamos alguns estudos:

Neres et al. (2017): "O aumento dos espaços intercristalinos presentes no esmalte das LMB leva a uma diminuição na sua microdureza”.

Taher et al. (2012): "ICON aumenta a microdureza do esmalte, pois este material é capaz de substituir a perda mineral por resina de baixa viscosidade o que possibilita a penetração do infiltrante no corpo da lesão".

Neres et al. (2017): Demonstrou diminuição da microdureza e aumento da rugosidade de superfície dental usando a técnica de infiltração com ICON nas Lesões de Mancha Branca (LMB).

Neres et al. (2017) e Gurdogan et al. (2017) apud Simão (2017): “A capacidade protetora da resina de baixa viscosidade parece aumentar significativamente a microdureza quando a infiltração é aplicada em duas camadas em vez de uma única aplicação, mesmo após exposição a um novo ambiente cariogênico".

Taher, Alkhamis \& Dowaidi (2012): “A polimerização a resina proporciona um aumento da microdureza do esmalte".

Borges et al. (2017), Neres et al. (2017) apud Simão (2017): Reportam que não há diferenças estatisticamente significativas entre a rugosidade superficial de dentes tratado com ICON e a rugosidade de dentes com esmalte.

Tendo como base os estudos acima, o Infiltrante Resinoso (ICON) aumenta a microdureza do esmalte, pois este penetra no corpo da lesão e quando polimerizado substitui o mineral perdido. Quanto à textura/rugosidade da superfície, o ICON não apresenta problema, pois a rugosidade por ele provocada é aceitável clinicamente.

No entanto, o polimento da lesão infiltrada é muito importante para a melhoria da estabilidade da cor, devido a diminuição da rugosidade de superfície e possível remoção da camada superficial de inibição de oxigênio (Cohen-Carneiro et al., 2014; Gurdogan, Ozdemir-Ozenen, \& Sandalli, 2017 apud Simão, 2017).

Além disso, há diversas dúvidas sobre a eficácia desse produto sobre as lesões de mancha branca no esmalte. Como exposto no trabalho, inúmeras alterações morfológicas são responsáveis pela aparição das indesejadas manchas brancas na superfície do esmalte. Sendo assim, alguns estudos foram feitos para comprovar a efetividade desse sobre as lesões de mancha branca ocasionada pela fluorose dentária, cárie dental associada a aparelhos ortodônticos e amelogênese imperfeita. Vejamos:

Attal et al. (2014) e Giannetti et al. (2018) apud Roselli (2019): “Casos em que a lesão, apesar da origem superficial, se desenvolve em profundidade, como nos casos de Hipomineralização de Incisivos e Molares $(\mathrm{MIH})$, das formas graves de fluorose e da hipomineralização traumática, o potencial de infiltração é rapidamente atingido e apenas uma pequena parte da lesão é infiltrada, com a consequência de um disfarce insuficiente da lesão".

Silva (2014): "Estudos clínicos randomizados comprova a eficácia da técnica de infiltração resinosa para tratamento de lesões interproximais, em dentes decíduos e permanentes e LMB vestibulares".

Neuhaus et al. (2013): "O controle das lesões de cárie com a técnica da infiltração resinosa se demonstra mais eficaz em lesões ativas quando comparados as lesões inativas. Isso ocorre devido a maior porosidade da superfície em lesões ativas, facilitando a penetração do infiltrante e o preenchimento do corpo da lesão".

Meyer-Lueckel et al. (2016): Avaliaram durante três anos 22 jovens e compararam a eficácia do tratamento das lesões proximais tratadas com ICON (grupo teste) com lesões proximais tratadas com placebo (grupo 
controle). A primeira avaliação após 18 meses da população do presente estudo revelou progressão de $7 \%$ e $37 \%$ das lesões do grupo teste e controle, respectivamente, não havendo diferenças até o período de 3 anos. Estes resultados sustentam a eficácia do ICON no impedimento da progressão das cáries proximais não cavitadas até ao terço externo da dentina.

Paris et al. (2014): Em estudo onde avalia a penetração do infiltrante (ICON) e de um selante, quando aplicados conforme recomendado, em lesões superficiais de mancha branca ICDAS 1 (Mancha branca/marrom no esmalte seco) e ICDAS 2 (Mancha branca/ marrom no esmalte molhado). Os resultados do tratamento das lesões com ICON para as lesões ICDAS 1 foram profundidade da lesão (PL) média de 271um e profundidade de infiltração (PI) média de 51um; para as lesões ICDAS 2 foram PL média de 538um e PI média de 186um. Simão (2017) evidenciou a capacidade do infiltrante resinoso ICON de travar a progressão de lesões codificadas com ICDAS 1 e ICDAS 2.

Borges et al. (2017): “O efeito de camuflagem das LMB parece ser influenciado pela extensão profundidade e atividade da cárie. Através da infiltração, o ICON não consegue penetrar numa superfície de lesão remineralizada e, consequentemente, a lesão cariogenicamente inativa não adquire o efeito da camuflagem, continuando com o mesmo aspecto esbranquiçado inicial".

Ribeiro, Oliveira e Araújo (2016): “As resinas de baixa viscosidade são contraindicadas, entretanto, para lesões que acometem o segundo terço da dentina ou toda a sua extensão (D3 e D4) e para áreas onde existe provável cavitação".

Denis et al. (2013), Giannetti et al. (2018a) e Giannetti et al. (2018b) apud Rosseli (2019): "Casos de hipomineralização traumática e no Hipomineralização de Incisivos e Molares (MIH) a infiltração pode ser incompleta devido a possibilidade da presença de um ângulo agudo, que leva a um contorno da lesão conhecido como "edge effect", no qual a erosão por si só não consegue remover as suspensões periféricas de esmalte saudável que impedem que a resina penetre na totalidade da lesão. Esses resultados inconsistentes significam que o tratamento deve ser realizado com a devida cautela, analisando o tipo de lesão e procurando modificações do protocolo para melhorar a eficácia”.

Bhandari et al. (2018): Avaliou o resultado estético numa lesão Hipomineralização de Incisivos e Molares $(\mathrm{MIH})$ leve através de um estudo in vivo, afirma que o tratamento da infiltração de resina, apesar de uma redução acentuada da cor, não mascara a lesão inteira.

Andrade (2019): “O tratamento com resina infiltrante não foi capaz de deixar a lesão de mancha branca do esmalte dental com a mesma coloração do tecido hígido. No entanto, o tratamento foi capaz de atenuar a descoloração do esmalte dentário desmineralizado".

Sant'anna et al. (2016): “A aplicação de ICON não reduziu completamente as lesões de mancha branca, mas o aspecto final apresentou-se satisfatório, com homogeneidade e brilho à superfície, o que mitigou os efeitos desagradáveis apresentados inicialmente”.

Logo, com base nos estudos mostrados, constata-se que o Infiltrante Resinoso (ICON), não é capaz de acabar com a mancha branca, mas pode camufla-la. Tal camuflagem é influenciada pela extensão, profundidade e atividade da cárie, ou seja, é mais indicado para lesão mais superficial E1, E2 e D1 (Fluorose leve, cárie no estágio inicial) e contraindicado para lesão profundas D2 e D3 (Hipomineralização de Incisivos e Molares (MIH) e fluorose dentária grave, lesão de cárie com cavitação). Também, mostrou resultados satisfatórios para impedir a progressão da cárie e mais eficaz para mimetizar as lesões de cárie ativa.

No mais, estudos mostram a vulnerabilidade do Infiltrante Resinoso (ICON) à pigmentação, vejamos:

Borges et al. (2017), Borges et al. (2014) e Rey et al. (2014) apud Simão (2017): "ICON possui uma grande susceptibilidade a pigmentação, quando comparado com outras metodologias".

Rey et al. (2014): Comparou o potencial de pigmentação do infiltrante resinoso ICON e diversos agentes adesivos em submersão de vinho, café e chá. Frente a todos os materiais testados o ICON apresentou o maior índice de pigmentação, sendo que o café foi o responsável pelos maiores índices, seguido pelo vinho e chá.

Borges et al. (2014): Demostrou que a imersão de espécimes tratados por infiltração resinosa em vinho, café e água destilada resultou significativa alteração de cor para as duas primeiras substâncias em comparação com a terceira e, apesar do repolimento minimizar o manchamento, os espécimes submersos em vinho e café ainda apresentaram taxa de pigmentação superiores à água destilada.

Cohen-Carneiro et al. (2014): Avaliaram a estabilidade de cor de lesões de cárie artificial em esmalte bovino tratado com infiltração de resina ou remineralização, após imersão em diferentes soluções (saliva, café e vinho tinto).

Simão (2017): Mostrou um resultado menos favorável para ICON no que se refere à estabilidade da coloração da LMB, quando comparadas as lesões remineralizadas. 
No entanto, conclui-se que o infiltrante resinoso (ICON) comparado a outros produtos adesivos, apresenta grande susceptibilidade a pigmentação. De acordo com Borges et al. (2017) a susceptibilidade à pigmentação do ICON pode estar relacionada com a maior taxa de absorção de água do produto.

\section{Considerações finais}

Pode-se concluir, portanto, que o Infiltrante Resinoso (ICON) é considerado um produto minimamente invasivo capaz de paralisar a lesão cariosa, aumentar a microdureza do esmalte e camuflar manchas brancas na superfície do esmalte dental. Contudo, sua eficácia é comprovada no tratamento de manchas brancas de cárie na face vestibular do dente, ocasionada pelo uso de aparelho ortodôntico e fluorose dentária leve. Entretanto, sua aplicação em áreas estéticas é questionada, pois este apresenta alta susceptibilidade de pigmentação exógena.

\section{Referência}

Akin, M., Tazcan, M., Ileri, Z., \& Ayhan, F. 2013. Incidence of white spot lesion during fixed orthodontic treatment. Turkish J Orthod Vol, 26(2), 98-102.

Andrade, R. M. P. M. B. 2019. Avaliação clínica de um infiltrante resinoso utilizado para mascarar lesões de mancha branca no esmalte dental. Dissertação de Mestrado. Uberaba, MG: Universidade de Uberaba.

Aoba, T., \& Fejerskov, O. 2002. Dental fluorosis: chemistry and biology. Critical Reviews in Oral Biology and Medicine, 13(2), 155-170.

Bath-balogh, M., \& Fehrenbach, M. J. 2012. Anatomia, histologia e embriologia dos dentes e das estruturas orofaciais. Rio de Janeiro, RJ: Elsevier.

Bevilacqua, F. M., Sacramento, T., \& Felício, C. M. 2010. Amelogênese imperfeita, hipoplasia de esmalte e fluorose dental - revisão da literatura. Revista Uniara, 13(2), 136-148.

Bhandari, R., Thakur, S., Singhal, P., Chauhan, D., Jayam, C., \& Jain, T. (2018). Concealment effect of resin infiltration on incisor of Grade I molar incisor hypomineralization patients: An in vivo study. Journal of Conservative Dentistry: JCD, 21(4), 450.

Bishara, S. E., \& Ostby, A. W. 2008. White Spot Lesions: Formation, Prevention, and Treatment. Seminars in Orthodontics, 14(3),174-182.

Borges, A. B., Caneppele, T. M. F., Luz, M., Pucci, C. R., \& Torres, C. R. G. 2014. Color stability of resin used for caries infiltration after exposure to different staining solutions. Operative dentistry, 39(4), 433-440.

Borges, A. B., Caneppele, T. M. F., Masterson, D., \& Maia, L. C. 2017. Is resin infiltration an effective esthetic treatment for enamel development defects and white spot lesions? A systematic review. Journal of dentistry, $56,11-18$.

Campos, D. L., Farias, D. G., \& Toledo, O. A. 1998. Prevalência de fluorose dentária em escolares de Brasília Distrito Federal. Revista de Odontologia da Universidade de São Paulo, 12(3), 225-230.

Chapman, J. A., Roberts, W. E., Eckert, G. J., Kula, K. S., \& González-Cabezas, C. 2010. Risk factors for incidence and severity of white spot lesions during treatment with fixed orthodontic appliances. American Journal of Orthodontics and Dentofacial Orthopedics, 138(2), 188-194.

Cohen-Carneiro, F., Pascareli, A. M., Christino, M. R. C., Vale, H. F. D., \& Pontes, D. G. 2014. Color stability of carious incipient lesions located in enamel and treated with resin infiltration or remineralization. International journal of paediatric dentistry, 24(4), 277-285.

Correia, R. T. 2012. Selamento de lesões de cárie proximal com infiltrante resinoso: estudo clinico randomizado. Dissertação de Mestrado. Santa Maria, RS: Universidade Federal de Santa Maria.

Dayila, J. M., Buonocore, M. G., Greeley, C. B., \& Provenza, D. V. (1975). Adhesive penetration in human artificial and natural white spots. Journal of Dental Research, 54(5), 999-1008.

Denbesten, P. K. 1999. Biological mechanisms of dental fluorosis relevant to the use of fluoride supplements. Community Dentistry and Oral Epidemiology, 27(1), 41-47.

Dias, T. R. 2015. Lesão de mancha branca associada ao tratamento ortodôntico fixo. Revisão de literatura. Trabalho de Conclusão de Curso. Brasília, DF: Universidade de Brasília.

Fejerskov, O., Thylstrup, A., \& Larsen, M. J. 1977. Clinical and structural features and possible pathogenic mechanisms of dental fluorosis. Scandinavian Journal of Dental Research, 85(7), 510-534. 
Gerlach, R. F., Sousa, M. L. R., de. \& Cury, J. A. 2000. Esmalte Dental com Defeitos: de marcador biológico a Implicações clínicas. Revista Odonto Ciência, 15(31), 87-102.

Gomes, D., \& Da Ros, M. A. 2008. A etiologia da cárie no estilo de pensamento da ciência odontológica. Ciência \& Saúde Coletiva, 13(3), 1081-1090.

Guedes-Pinto, A. C., \& Mello-Moura, A. C. V. 2016. Odontopediatria (9a ed.). Rio de Janeiro, RJ: Santos.

Katchburian, E., \& Arana, V. 1996. Histologia e embriologia oral (1a ed.). Rio de Janeiro, RJ: Guanabara Koogan.

Mangueira, D. F. B., Passos, I. A., Pereira, A. M. B. C., \& Oliveira, A. F. B. D. 2011. Cárie e erosão dentária: uma breve revisão. Odontologia Clínico-Científica, 10(2), 121-124.

Marsh, P. D. O. 1995. Papel da microbiologia em modelos de cárie dentária. Avanços na pesquisa odontológica, 9(3), 244-254.

Mazur, M., Westland, S., Guerra, F., Corridore, D., Vichi, M., Maruotti, A., ... \& Ottolenghi, L. 2018. Objective and subjective aesthetic performance of icon ${ }^{\circledR}$ treatment for enamel hypomineralization lesions in young adolescents: A retrospective single center study. Journal of dentistry, 68, 104-108.

Melo, P., Teixeira, L., \& Domingues, J. 2006. A importância do despiste precoce de cárie dentária. Revista Portuguesa de Medicina Geral e Familiar. Porto, 22(3), 357-366.

Menezes, L. M. B. D., Sousa, M. D. L. R. D., Rodrigues, L. K. A., \& Cury, J. A. 2002. Autopercepção da fluorose pela exposição a flúor pela água e dentifrício. Revista de Saúde Pública, 36, 752-754.

Meyer-Luckel, H., \& Paris, S. 2009. Masking of labial enamel white spot lesions by resin infiltration - a clinical report. Quintessence International, 40(9), 713-718.

Meyer-Lueckel, H., Balbach, A., Schikowsky, C., Bitter, K., \& Paris, S. (2016). Pragmatic RCT on the efficacy of proximal caries infiltration. Journal of Dental Research, 95(5), 531-536.

Min, J. H., Inaba, D., Kwon, H. K., Chung, J. H., \& Kim, B. I. (2015). Evaluation of penetration effect of resin infiltrant using optical coherence tomography. Journal of Dentistry, 43(6), 720-725.

Moffatt, P., Smith, C. E., St-Arnaud, R., \& Nanci, A. 2008. Characterization of Apin, a secreted protein highly expressed in tooth-associated epithelia. Journal of Cellular Biochemistry, 103(3), 941-956.

Neres, É. Y., Moda, M. D., Chiba, E. K., Briso, A. L. F., Pessan, J. P., \& Fagundes, T. C. 2017. Microhardness and roughness of infiltrated white spot lesions submitted to different challenges. Operative dentistry, 42(4), 428-435.

Neuhaus, K. W., Schlafer, S., Lussi, A., \& Nyvad, B. 2013. Infiltration of natural caries lesions in relation to their activity status and acid pretreatment in vitro. Caries research, 47(3), 203-210.

Neville, B. W., \& Damm, D. D. 2009. Patologia oral e maxilofacial (3a ed.). Rio de Janeiro, RJ: Elsevier.

Newman, M. G. 2007. Carranza, Periodontia Clínica (10a ed.). Rio de Janeiro, RJ: Saunders Elsevier.

Øgaard, B. 2008. White Spot Lesions During Orthodontic Treatment: Mechanisms and Fluoride Preventive Aspects. Seminars in Orthodontics, 14(3), 183-193.

Oguro, R., Nakajima, M., Seki, N., Sadr, A., Tagami, J., \& Sumi, Y. 2016. The role of enamel thickness and refractive index on human tooth colour. Journal of dentistry, 51, 36-44.

Olympio, K. P. K., Bardal, P. A. P., Henriques, J. F. C., \& Bastos, J. R. D. M. 2006. Prevenção de cárie dentária e doença periodontal em Ortodontia: uma necessidade imprescindível. Revista Dental Press de Ortodontia e Ortopedia Facial, 11, 110-119.

Paris, S., Meyer-Lueckel, H., Cölfen, H., \& Kielbassa, A. M. 2007. Resin infiltration of artificial enamel caries lesions with experimental light curing resins. Dental materials journal, 26(4), 582-588.

Paris, S., Lausch, J., Selje, T., Dörfer, C. E., \& Meyer-Lueckel, H. 2014. Comparison of sealant and infiltrant penetration into pit and fissure caries lesions in vitro. Journal of dentistry, 42(4), 432-438.

Passos, I. A., Costa, J. D. M. C., Melo, J. M., Forte, F. D. S., \& Sampaio, F. C. 2007. Defeitos do esmalte: etiologia, características clínicas e diagnóstico diferencial. Journal of the Health Sciences Institute, 187-192.

Quirino, A., Neto, V., Nobre, C., Lima, H., \& Feitosa, V. 2017. Tratamento de manchas por fluorose com utilização de infiltrante resinoso: relato de caso. Jornada Odontológica dos Acadêmicos da Católica, 2(1).

Rahiotis, C., Zinelis, S., Eliades, G., \& Eliades, T. 2015. Setting characteristics of a resin infiltration system for incipient caries treatment. Journal of dentistry, 43(6), 715-719.

Rey, N., Benbachir, N., Bortolotto, T., \& Krejci, I. 2014. Evaluation of the staining potential of a caries infiltrant in comparison to other products. Dental materials journal, 33(1), 86-91.

Ribeiro, L. S. M. V., Oliveira, C. T. P., \& Araújo, L. S. N. 2016. Infiltrantes e suas aplicações. Revista interfaces saúde, humanas e tecnologia, 3(9), 75-80. 
Roselli, F. 2019. Resina infiltrante como tratamento estético de manchas brancas: um caso clínico. Relatório Final de Estágio de Mestrado. Gandra: Instituto Universitário de ciências da saúde.

Sant'anna, G. R. D., Silva, I. M., Lima, R. L., Souza-Zaroni, W. C., Leite, M. F., \& Samiei, M. (2016). Infiltrante resinoso vs Microabrasão no manejo de lesões de mancha branca: relato de caso. Revista da associação Paulista de cirurgiões dentistas, 70(2), 187-197.

Sharawy, M., \& Yaeger, J. A. 1989. Esmalte. In: Braskar SN. Histologia e Embriologia de Orban. São Paulo, SP: Artes Médicas.

Silva, M, B. 2014. Avaliação da remoção de esmalte superficial, da capacidade de mascaramento de manchas brancas e da resistência à pigmentação extrínseca das técnicas de microabrasão e infiltração resinosa. Dissertação de Mestrado Brasília, DF: Universidade de Brasília.

Simão, A. A. T. 2017. Influência da infiltração de resina (icon) ${ }^{\circledR}$ nas lesões de mancha branca. Artigo de Revisão Bibliográfica apresentado ao mestrado. Porto: Faculdade de Medicina Dentária.

Subtil, B., Proença, L., Polido, M., Azul, A. C., \& Moura, P. D. M. 2016. Microdureza do esmalte após tratamento com a técnica de microabrasão e com ICON ${ }^{\circledR}$. In XXXVI Congresso Anual SPEMD (Sociedade Portuguesa de Estomatologia e Medicina Dentária) (Vol. 57, p. 33). Sociedade Portuguesa de Estomatologia e Medicina Dentária.

Taher, N. M., Alkhamis, H. A., \& Dowaidi, S. M. 2012. The influence of resin infiltration system on enamel microhardness and surface roughness: An in vitro study. The Saudi Dental Journal, 24(2), 79-84.

Takashino, N., Nakashima, S., Shimada, Y., Tagami, J., \& Sumi, Y. 2016. Effect of thermal cyclic stress on acid resistance of resin-infiltrated incipient enamel lesions in vitro. Dental materials journal, 35(3), 425-431.

Thylstrup, A., \& Fejerskov, O. 1978. Clinical appearance of dental fluorosis in permanent teeth in relation to histologic changes. Community Dentistry and Oral Epidemiology, 6(6), 315- 328.

Tong, L. S. M., Pang, M. K. M., Mok, N. Y. C., King, N. M., \& Wei, S. H. Y. 1993. The effects of etching, microabrasion, and bleaching on surface enamel. Journal of dental research, 72(1), 67-71.

Torres, C. R. G., Rosa, P. C. F., Ferreira, N. S., \& Borges, A. B. 2012. Effect of caries infiltration technique and fluoride therapy on microhardness of enamel carious lesions. Operative dentistry, 37(4), 363-369.

Tumenas, I., Pascottos, R., Saade, J. L., \& Bassani, M. 2014. Odontologia minimamente invasiva. Revista da Associacao Paulista de Cirurgioes Dentistas, 68(4), 283-295.

Ulrich, I., Mueller, J., Wolgin, M., Frank, W., \& Kielbassa, A. M. 2015. Tridimensional surface roughness analysis after resin infiltration of (deproteinized) natural subsurface carious lesions. Clinical oral investigations, 19(6), 1473-1483.

Vellasco, K., Campos, I., Ferreira, T. D. R. F. Z., \& Basting, R. T. 2006. Dentística minimamente invasiva: plástica dental. Arquivos em Odontologia, 42(2).

Zaitouni, F. 2014. Abordagem Clínica Da Erosão Dentária. Dissertação de mestrado. Porto: Universidade Fernando Pessoa.

\section{Minicurrículo}

Danieli Dias. Graduanda em Odontologia pela UNILESTE-MG.

Como citar: Dias, D. 2021. Uso de infiltrante resinoso (ICON) para tratamento de lesões de mancha branca. Pubsaúde, 7, a234. DOI: https://dx.doi.org/10.31533/pubsaude7.a234 Recebido: 19 jul. 2021.

Revisado e aceito: 3 ago. 2021.

Conflito de interesse: os autores declaram, em relação aos produtos e companhias descritos nesse artigo, não ter interesses associativos, comerciais, de propriedade ou financeiros que representem conflito de interesse.

Licenciamento: Este artigo é publicado na modalidade Acesso Aberto sob a licença Creative Commons Atribuição 4.0 (CC-BY 4.0). 